\title{
Can ecotourism change community attitudes towards conservation?-CORRIGENDUM
}

DOI: 10.1017/S0030605319000607. Published online by Cambridge University Press, 5 May 2020

Table 3 was incorrect in this Article. The correct table is provided here.

\section{Reference}

Ziegler, J., Araujo, G., Labaja, J., Snow, S., King, J.N., Ponzo, A. et al. (2020) Can ecotourism change community attitudes towards conservation? Oryx, published online 5 May 2020.

TABLE 3 Conservation outcomes of whale shark tourism activities at the four tourism sites, as reported in $\mathrm{n}$ interviews at each site.

\begin{tabular}{|c|c|c|c|c|c|c|c|}
\hline Conservation outcomes & $\begin{array}{l}\text { 1. Mass } \\
\text { (Oslob, } \\
\mathrm{n}=25 \text { ) }\end{array}$ & $\begin{array}{l}\text { 2. Mid-tier } \\
\text { (Donsol, } \\
\mathrm{n}=24 \text { ) }\end{array}$ & $\begin{array}{l}\text { 3. Small } \\
\text { (Pintuyan, } \\
\mathrm{n}=40 \text { ) }\end{array}$ & $\begin{array}{l}\text { 4. Failed } \\
\text { (Talisayan, } \\
\mathrm{n}=25 \text { ) }\end{array}$ & $\begin{array}{l}\text { Test } \\
\text { statistic }\end{array}$ & $\mathrm{P}$ & $\begin{array}{l}\text { Effect } \\
\text { size }\end{array}$ \\
\hline \multicolumn{8}{|l|}{ Changes in fishing $^{1}$} \\
\hline $\begin{array}{l}\text { \% fishers for whom tourism } \\
\text { has changed the amount of } \\
\text { fishing they do (n) }\end{array}$ & $87.5(14)$ & $40.9(9)$ & $19.4(7)$ & & $\chi^{2}=21.285$ & $<0.001^{\star}$ & 0.467 \\
\hline $\begin{array}{c}\text { Mean } \pm \text { SE } \% \text { change in fishing } \\
\text { due to whale shark tourism }\end{array}$ & $-54.9 \pm 7.85^{\mathrm{a}}$ & $-66.7 \pm 7.55^{\mathrm{a}}$ & $-5.7 \pm 16.42^{\mathrm{b}}$ & & $\mathrm{F}=8.259$ & $0.002^{*}$ & 0.616 \\
\hline $\begin{array}{l}\text { \% fishers who changed from fishing } \\
\text { to tourism as main source of } \\
\text { income }(\mathrm{n})\end{array}$ & $92.3(12)$ & $31.8(7)$ & $0.0(0)$ & & $\chi^{2}=22.944$ & $<0.001^{\star}$ & 0.706 \\
\hline \multicolumn{8}{|c|}{ Changes in attitudes towards whale sharks } \\
\hline $\begin{array}{l}\% \text { respondents who changed } \\
\text { their view of whale sharks }\end{array}$ & 72.0 & 95.8 & 95.0 & 52.0 & $\chi^{2}=23.218$ & $<0.001^{\star}$ & 0.451 \\
\hline $\begin{array}{l}\% \text { respondents who like } \\
\text { whale sharks }\end{array}$ & 100.0 & 100.0 & 100.0 & 76.0 & $\chi^{2}=22.547$ & $<0.001^{*}$ & 0.445 \\
\hline $\begin{array}{l}\% \text { respondents who believe } \\
\text { whale sharks are an important } \\
\text { animal in the Philippines }\end{array}$ & 100.0 & 100.0 & 100.0 & 44.0 & $\chi^{2}=56.818$ & $<0.001^{\star}$ & 0.706 \\
\hline $\begin{array}{l}\text { \% respondents who believe } \\
\text { whale sharks should be protected } \\
\text { from being killed }\end{array}$ & 100.0 & 100.0 & 100.0 & 16.0 & $\chi^{2}=91.641$ & $<0.001^{*}$ & 0.897 \\
\hline $\begin{array}{l}\text { \% respondents who believe } \\
\text { the Philippines will change } \\
\text { if whale sharks go extinct }\end{array}$ & 64.0 & 70.8 & 87.5 & 8.0 & $\chi^{2}=42.550$ & $<0.001^{\star}$ & 0.611 \\
\hline \multicolumn{8}{|l|}{ Changes in behavioural intentions } \\
\hline $\begin{array}{l}\text { Mean } \pm \text { SE score for desire to } \\
\text { protect whale sharks }\end{array}$ & $4.0 \pm 0.04^{\mathrm{a}}$ & $4.0 \pm 0.00^{\mathrm{a}}$ & $4.0 \pm 0.04^{\mathrm{a}}$ & $2.9 \pm 0.23^{b}$ & $\mathrm{~F}=23.187$ & $<0.001^{\star}$ & 0.624 \\
\hline $\begin{array}{l}\text { Mean } \pm \text { SE score for desire to } \\
\text { protect ocean }\end{array}$ & $3.8 \pm 0.10^{\mathrm{a}}$ & $4.0 \pm 0.00^{\mathrm{a}, \mathrm{b}}$ & $3.9 \pm 0.05^{\mathrm{a}, \mathrm{b}}$ & $3.3 \pm 0.17^{\mathrm{a}, \mathrm{c}}$ & $F=9.524$ & $<0.001^{\star}$ & 0.456 \\
\hline \multicolumn{8}{|l|}{ Changes in behaviours } \\
\hline $\begin{array}{l}\text { \% respondents who changed } \\
\text { their behaviour to protect } \\
\text { whale sharks }\end{array}$ & 48.0 & 75.0 & 92.5 & 44.0 & $\chi^{2}=22.940$ & $<0.001^{\star}$ & 0.449 \\
\hline $\begin{array}{l}\% \text { respondents who changed } \\
\text { their behaviour to protect } \\
\text { the ocean }\end{array}$ & 64.0 & 79.2 & 95.0 & 64.0 & $\chi^{2}=12.495$ & $0.006^{\star}$ & 0.331 \\
\hline
\end{tabular}

Post-hoc tests were calculated for ANOVA results using least significant difference if equal variance assumed (i.e. if Levene statistic $\mathrm{P}>0.05$ ) and Games-Howell if equal variances not assumed (i.e. if Levene's statistic $\mathrm{P}<0.05$ ); normal distribution of data not tested because it does not affect the outcome of parametric tests (Vaske, 2008); means with different superscript letters in the same row are significantly different $(P<0.05)$.

${ }^{\star}$ Significant at $\alpha=0.05$.

${ }^{1}$ The percentages reported in this section are not based on the total sample size for each site but the number of respondents who were fishers at each site (Donsol: $\mathrm{n}=19$, Oslob: $\mathrm{n}=15$, Pintuyan: $\mathrm{n}=34$, Talisayan: $\mathrm{n}=21$ ). 